\title{
UNA VISIÓN DIDÁCTICA DE RIESGO VERSUS INCERTIDUMBRE
}

\author{
Elisa A. González del Valle Campoamor* \\ Departamento de Economía, Universidad Autónoma Metropolitana-Iztapalapa
}

\author{
Ma. Cristina Escobar Iturbe \\ Departamento de Economía,Universidad Autónoma Metropolitana-Iztapalapa \\ (Recibido 20 de enero 2003, aceptado 28 de agosto 2003)
}

\begin{abstract}
Resumen
En este trabajo, el significado de las palabras "riesgo" e "incertidumbre" será explicada así como los métodos capaces de incorporarlos en el análisis económico. El énfasis se ha concentrado en el riesgo incorporado en el proceso de planeación del presupuesto. La desviación típica es la medida de riesgo más común usada en la literatura económica y financiera, la cual mide la dispersión alrededor de la media de una distribución de resultados posibles. El coeficiente de variación, el cual establece la desviación típica sobre una base relativa, ayuda a comparar proyectos con valores presentes netos esperados desiguales. El cálculo del valor presente neto y de la desviación para proyectos multi-periodo, bajo condiciones de independencia estadística, también es discutido. En la realidad, estos cálculos no se emplean debido a su complejidad. En su lugar, la tasa de descuento se ajusta con frecuencia para incorporar el riesgo. La tasa de descuento riesgo-ajustado (RADR) es una técnica dominante en los negocios.
\end{abstract}

\begin{abstract}
In this study, the meaning of the words "risk" and "uncertainty" will be explained as well as the various methods avilable to incorporate them into economic analysis. The emphasis has been focused on incorporating risk into capital budgeting process. The most common measure of risk in economic and financial literature is the standard deviation, which measures the dispersion around the mean of a distribution of possible outcomes. The coefficient of variation, which puts the standard deviation on a relative basis, helps in comparing projects with unequal expected net present values. The calculation-of the expected net present value and deviation for multi-period projects under conditions of statistical independence is also discussed. On real business situations, these computing are often not employed because of their complexity. Instead, the discount rate is frequently adjusted to incorporate risk. The risk-adjusted discount rate (RADR) is a dominant technique in business.
\end{abstract}

Clasificación JEL: D81

Palabras clave: Riesgo, Incertidumbre

* Universidad Autónoma Metropolitana-Iztapalapa, Departamento de Economía. San Rafael Atlixco No. 186, Col. Vicentina, Del. Igtapalapa, 09340, México, D. F. Teléfono: $+52(55) 58044768$.

Los autores desean agradecer a dos dictaminadores anónimos sus valiosas observaciones. 


\section{Introducción}

Tanto en teoría financiera como en análisis económico, los términos de riesgo e incertidumbre tienen en ocasiones diferente significados aunque son usados en forma indistinta. A pesar de que ciertos eventos no ocurren con certeza, existen condiciones que permiten asignar probabilidades a eventos por ocurrir, mientras que a otros no. Cuando es posible asignar una probabilidad a algún evento futuro se tiene el caso de riesgo. Por ejemplo, en una compañía se estiman ventas $^{1}$ por $\$ 5,000,000$ de un producto para el próximo año con una probabilidad del $25 \%$, de $\$ 6,000,000$ con una probabilidad de $50 \%$ y de $\$ 7,000,000$ con una de $25 \%$. Cuando no existe forma de asignar probabilidades de ocurrencia a eventos futuros, entonces se tiene una situación de incertidumbre.

Antè de discutir como el riesgo es tratado en análisis económico, es conveniente explicar por qué las empresas enfrentan condiciones de riesgo en el mundo de los negocios. En primer lugar, se tienen las condiciones del sistema económico, las empresas enfrentan ciclos de crecimiento y decrecimiento en los negocios. Algunos sucesos se pueden abordar utilizando técnicas de pronóstico acerca de las fluctuaciones económicas, pero nunca pueden ser conocidos con certeza. Es más, el efecto en general de la actividad económica en una empresa o producto específico no es conocido con anticipación. Por lo tanto, una empresa no está completamente preparada para afrontar estos cambios en la actividad económica. En suma, la incertidumbre con respecto a la economía es tal que las fiuctuaciones relacionadas con los negocios no siempre coinciden con los cambios de la economía en su conjunto.

Por ejemplo, la competencia de una firma no siempre es conocida con certeza. Existe una relación estrecha entre las acciones realizadas por la conpetencia y los cambios en la tecnología correspondiente. Si un competidor dentro de cierta industra introduce en el mercado un bien o servicio innovador, las ventas pueden ser fuertemente afectadas. Por ejemplo, la introducción del correo electrónico impactó drásticamente el mercado de las máquinas de fax siendo sustituidos de forma casi inmediata.

En segundo lugar, los cambios en la demanda de los consumidores son otra causa de riesgo para las empresas. Un producto exitoso en un año o estación puede ser no requerido el siguiente periodo. Tercero, no todas las fuentes de incertidumbre con respecto a los cambios en la demanda son debidos al consumidor. En el proceso de toma de decisiones, una compañía también debe considerar costos y gastos; cuando se realiza una estimación de gastos, la empresa no puede estar segura de que los precios de los factores se mantengan (excepto si estos son pactados de antemano con los proveedores); de esta manera, los precios de los insumos pueden cambiar, así como los costos de la electricidad y otros servicios. Los costos del trabajo también pueden estar sujetos a fluctuaciones, a menos que hayan sido contratados.

Es fácil ver que la mayoría de los eventos futuros en los negocios son inciertos. Ambos factores, costos y rentas, son inciertos a lo largo de toda la vida del bien o servicio. Obviamente, esto complica el trabajo de las empresas. Sin embargo, esto también las obliga a reducir considerablemente el riesgo en el

1 Utilizando datos históricos del comportamiento de las ventas y/o incorporando el juicio de expertos del mercado correspondiente. 
futuro a través de mejores políticas de control y de toma de decisiones. Por lo tanto, el diseño de políticas de control en la administración es un área de importancia en las empresas.

\section{Medidas de riesgo}

Una vez presentadas algunas de las diferentes causas de riesgo en el mundo de los negocios, conviene analizar algunas medidas de riesgo. Primero, si existe un número de posibles resultados y cada uno de ellos tiene cierta probabilidad de ocurrencia (por ejemplo, en las estimaciones futuras de flujo de efectivo, el analista debe decidir la probabilidad para cada uno de los posibles resultados) entonces es posible construir una función de distribución de probabilidad. Esta función describe, en términos de porcentajes, la posibilidad de ocurrencia de todos los posibles resultados. Cuando se realiza la suma de todas las probabilidades de ocurrencia, ésta debe ser igual a uno, debido a que la probabilidad de ocurrencia de la unión de todos los eventos dentro del espacio muestral siempre ocurre con certeza. De esta forma, se puede realizar la asignación de probabilidades a los diferentes flujos de efectivo, como se muestra en el cuadro 1.

Cuadro 1. Distribución de probabilidad de flujos de efectivo.

\begin{tabular}{|c|c|}
\hline Flujo de efectivo (\$) & Probabilidad \\
\hline 3000 & 0.1 \\
\hline 4000 & 0.2 \\
\hline 5000 & 0.4 \\
\hline 6000 & 0.2 \\
\hline 7000 & 0.1 \\
\hline
\end{tabular}

La interpretación de la distribución de probabilidad para flujos de efectivo es la siguiente: Se estima que los posibles flujos de efectivo durante el siguiente año serán de $\$ 3,000.00, \$ 4,000.00, \$ 5,000.00, \$ 6,000.00$ y $\$ 7,000,00$. La ocurrencia de los cinco posibles flujos de dinero dependerá, por ejemplo, de las condiciones generales de la economía, las condiciones de la industria y de las acciones de la competencia. Si todos los factores son favorables al proyecto, el flujo de dinero será de $\$ 7,000.00$, si todos los factores son desfavorables, el Alujo de dinero será únicamente de $\$ 3,000.00$. Otros posibles flujos de dinero puede ocurrir si alguno de los factores trabaja a favor del proyecto o no.

La asignación de probabilidades indica que solamente hay un $10 \%$ de posibilidades para que todas las condiciones sean desfavorables y suceda la estimación mas baja del flujo de dinero. Igualmente, se tiene un $10 \%$ de posibilidades para que todas las condiciones sean favorables y suceda el máximo flujo de efectivo. Es mucho más probable que ocurran condiciones favorables y desfavorables al proyecto al mismo tiempo, por lo tanto, la ocurrencia de un flujo de dinero indeterminado es mayor. Una vez que se ha establecido la función de distribución de probabilidad, podemos calcular las dos medidas de riesgo utilizadas en la toma de decisiones.

Valor esperado: Tomando la función de distribución de probabilidad construída para los flujos de dinero es posible calcular el valor esperado de los posibles 
resultados. El valor esperado es simplemente el promedio de todos los posibles resultados ponderados por sus respectivas probabilidades.

$$
\bar{R}=\sum_{i=1}^{n} R_{i} p_{i}
$$

donde $\bar{R}=$ valor esperado, $R_{i}=$ valor en el caso $i, P_{i}=$ probabilidad en el caso i y $N=$ número de posibles resultados.

Gráfica 1. Distribución de probabilidad de los flujos de efectivo.

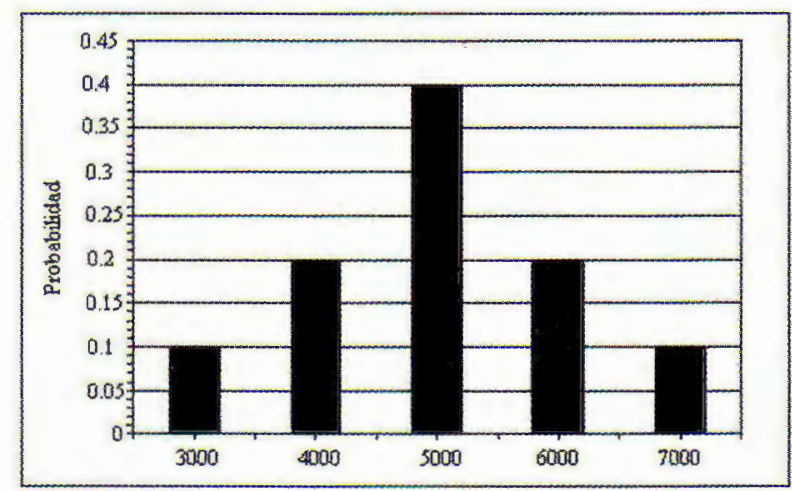

Una vez obtenido el promedio ponderado (valor esperado) es posible determinar una segunda medida de riesgo.

Desviación típica: En economía y finanzas, el riesgo es considerado como la dispersión de los posibles resultados alrededor del valor esperado. A mayor dispersión con respecto al promedio, el riesgo es mayor. De esta manera, para medir el riesgo es necesario encontrar un rango que refleje la variación de los posibles resultados respecto del promedio. La desviación típica es la raíz cuadrada de las desviaciones al cuadrado, respecto al promedio, de todos los posibles resultados.

$$
\sigma=\sqrt{\sum_{i=1}^{n}\left(R_{i}-\bar{R}\right)^{2} p_{i}}
$$

Para el ejemplo de los flujos de efectivo se tiene:

Cuadro 2.

\begin{tabular}{|c|c|c|c|}
\hline$R_{i}$ & $p_{i}$ & $\left(R_{i}-\bar{R}\right)$ & $\left(R_{i}-\bar{R}\right)^{2} p_{i}$ \\
\hline 3000 & .1 & -2000 & 400000 \\
\hline 4000 & .2 & -1000 & 200000 \\
\hline 5000 & .4 & 0 & 0 \\
\hline 6000 & .2 & 1000 & 200000 \\
\hline 7000 & .1 & 2000 & 400000 \\
\hline
\end{tabular}




$$
\sigma=\sqrt{120000}=1095
$$

¿Cuál es el significado de una desviación típica igual a 1095? Primero, la distribución de probabilidad de los flujos de efectivo es simétrica, esto significa que el $50 \%$ de la oportunidad de ocurrencia de los flujos de efectivo es mayor que el valor esperado, y que el $50 \%$ de la oportunidad de ocurrencia de los posibles flujos de efectivo es menor que el valor esperado. La oportunidad de ocurrencia de un flujo de efectivo en particular, depende de que tan grande es la desviación típica. Los flujos de efectivo describen una distribución normal aproximadamente, por lo cual podemos decir que $33 \%$ de todos los posibles flujos de efectivo se encuentran a una desviación típica respecto del promedio, el $47.7 \%$ de todos los flujos de efectivo se encuentran a dos desviaciones típicas del promedio y el $49.9 \%$ se encuentra a tres. De esta forma, dado que el valor esperado de los flujos de efectivo es $\$ 5,000.00$ y la desviación estándar es 1095 , se calculan los siguientes intervalos: $(\bar{R}-n \sigma)_{n=1,2,3}$. Su interpretación es la siguiente.

Existe un $33 \%$ de probabilidad para que los flujos de efectivo sean entre $\$ 5,000.00$ y $\$ 3,905.00$, en otras palabras existe un $16 \%$ de probabilidad de que el flujo de efectivo sea menor o igual a $\$ 3,905.00$. Más aún, con dos desviaciones (2190), existe un $2.3 \%$ de probabilidad $(50 \%-47.7 \%)$ de que el flujo sea igual o menor a $\$ 2,810.00$. Finalmente, tendremos la certeza de que ningún flujo de efectivo será menor a $\$ 1,715(5000-3 \sigma)$. Por este mismo razonamiento, podemos concluir que ningún flujo de efectivo será mayor a $\$ 8,285.00$ y existe $16 \%$ de probabilidad de que un flujo sea mayor de $\$ 6,095.00$.

\section{Distribuciones continuas}

La combinación entre valor esperado y desviación típica, permite que las empresas tomen decisiones entre dos proyectos diferentes. Supongamos que se tiene que seleccionar entre el proyecto $\mathrm{A}$ (flujo de efectivos con los parámetros obtenidos) y el proyecto B. Además, supongamos que ambos tienen el mismo valor esperado. La decisión se tomará con base en la desviación típica. El proyecto B, con mayor desviación estándar, es el de mayor riesgo. Generalmente, las empresas son adversas al riesgo, por lo tanto el proyecto $\mathrm{A}$, el cual tiene menor riesgo, será usualmente aceptado.

Las distribuciones de frecuencias de los proyectos discutidos son conocidas como distribuciones discretas. Los posibles resultados han sido limitados a cinco eventos para cada uno de los proyectos; sin embargo, es probable que otros flujos de efectivo ocurran (por ejemplo $\$ 4,679.00$ ó $\$ 6,227.00$ ). Si se consideran todos los posibles resultados, entonces se tiene una distribución continua. Los posibles resultados de una distribución continua son frecuentemente modelados por una curva en forma de campana, conocida como la curva normal. Es únicamente en este tipo de curva donde las propiedades referentes a la desviación típica mencionadas anteriormente, se cumplen estrictamente. Las dos curvas de los proyectos A y B tienen como centro al valor esperado, son simétricas y asintóticas.

Para cada uno de los proyectos se puede trazar una distribución normal de probabilidad, el proyecto A está modelado por una curva más ceñida al valor esperado (los valores están más concentrados alrededor del promedio ponderado), mientras que en el proyecto $\mathrm{B}$ los valores se encuentran más alejados del 
valor esperado. La desviación típica en el proyecto A es igual a 1095, entonces el $33 \%$ del área bajo la curva se encuentra entre $\$ 3,905.00$ y $\$ 5,000.00$. Para el proyecto B, la desviación típica es de 1710 y el $34 \%$ del área bajo la curva se encuentra entre $\$ 5,000.00$ y $\$ 3,290.00$.

La probabilidad de cualquier otro rango de valores puede ser fácilmente obtenida, utilizando la tabla de áreas bajo la curva de una distribución normal estándar. Por ejemplo, si en el proyecto A se está interesado en encontrar la probabilidad de que el Hujo de efectivo se encuentre por ejemplo entre $\$ 3,200.00$ y $\$ 5,000.00$, se aplica la transformación de estandarización a la variable aleatoria flujos de efectivo:

$$
Z=\frac{X-\bar{R}}{\sigma}
$$

donde $Z$ es el número de desviaciones típicas respecto a la media y $X$ es la variable de interés. Así, en el caso de los Flujos de efectivo se estandariza utilizando los parámetros del proyecto A, y se obtiene:

$$
Z=\frac{3200-5000}{1095}=\frac{-1800}{1095}=-1.64
$$

El valor 3200 es igual a 1.64 desviaciones típicas debajo de la media. Utilizando la tabla de áreas bajo la curva normal de media cero y desviación estándar uno, y leyendo en el renglón igual a 1.64, se encuentra el área bajo la curva igual a 0.4495 . Esto significa que la probabilidad de que el flujo de efectivo se encuentre entre $\$ 3,200.00$ y $\$ 5,000.00$ es del $45 \%$. Ya que en la curva normal ocurre que la mitad izquierda representa el $50 \%$ de todas las probabilidades, entonces se puede afirmar que la probabilidad de que un flujo de efectivo sea menor de $\$ 3,200.00$ es del $5 \%$.

Cuando el valor esperado de dos proyectos son iguales o parecidos, la desviación típica es una medida adecuada del riesgo. Se dice que la desviación típica es una medida absoluta del riesgo y por lo cual no cumple con el propósito de selección entre dos o más proyectos, cuando estos tienen valores esperados muy distintos. Por ejemplo, consideremos los siguientes proyectos con los siguientes parámetros:

\section{Cuadro 3.}

\begin{tabular}{|c|c|c|}
\hline & Valor esperado & Desviación típica \\
\hline Proyecto I & 100 & 30 \\
\hline Proyecto II & 50 & 20 \\
\hline
\end{tabular}

Ambos proyectos tienen valores esperados distintos y el valor absoluto del riesgo no responde adecuadamente la pregunta de selección o decisión entre ellos. En tal caso, un nuevo concepto es introducido, el coeficiente de variación, el cual mide el riesgo relativo del valor esperado. El coeficiente de variación es el cociente entre la desviación típica y el valor esperado:

$$
C V=\frac{\sigma}{\bar{R}}
$$


Entonces, para cada uno de los proyectos se obtiene:

Cuadro 4.

\begin{tabular}{|c|c|}
\hline Proyecto & Coeficiente de Variación \\
\hline I & $30 / 100=0.30$ \\
\hline II & $20 / 50=0.40$ \\
\hline
\end{tabular}

El coeficiente de variación del proyecto II es mayor. Así que el proyecto I aunque tiene una desviación típica mayor, su riesgo relativo es menor. De esta forma, el proyecto I es preferible al proyecto II, el cual tiene menos desviación típica, pero un riesgo relativo mayor.

El coeficiente de variación proporciona una solución satisfactoria para este tipo de toma de decisión. Sin embargo, el valor esperado, la desviación típica y el coeficiente de variación están limitados para su uso a un sólo periodo de decisión. En la mayoría de los casos, el riesgo tiene que ser medido a lo largo de varios periodos o años. La pregunta central es: ¿cómo tratar un capital, con propósito de inversión, en el cual el desembolso inicial promete retornar flujos de capital en un periodo futuro de varios años?

La primera tarea es calcular el valor presente neto de los valores esperados en cada periodo o año. Para un proyecto a tres años con una inversión inicial, el valor presente neto esperado es igual a:

$$
\overline{\mathrm{NPV}}=\frac{\bar{R}_{1}}{1+r_{j}}+\frac{\bar{R}_{2}}{\left(1+r_{j}\right)^{2}}+\frac{\bar{R}_{3}}{\left(1+r_{j}\right)^{3}}-Q_{0}
$$

o en términos generales:

$$
\overline{\mathrm{NPV}}=\sum_{t=1}^{n} \frac{\bar{R}_{t}}{\left(1+r_{f}\right)^{t}}-Q_{0},
$$

donde $\overline{\mathrm{NPV}}$ es el valor presente neto esperado, $\bar{R}_{t}$ es el valor esperado anual del flujo de efectivo, $Q_{0}$ es la inversión inicial y $R_{f}$ es la tasa libre de riesgo.

Cuadro 5 .

\begin{tabular}{|c|c|c|c|c|c|}
\hline \multicolumn{5}{|c|}{ Proceso de planeación del presupuesto bajo riesgo } \\
\hline & Año cero & Año uno & \multicolumn{2}{|c|}{ Año dos } \\
\hline & inversión inicial & $p$ & $R_{1}$ & $p$ & $R_{2}$ \\
\hline & -500 & .2 & 300 & .25 & 400 \\
\hline & & .6 & 500 & .50 & 500 \\
\hline & & .2 & 700 & .25 & 600 \\
\hline $\bar{R}_{t}$ & & & 500 & & 500 \\
\hline$r_{f}=0.05$ & \multicolumn{5}{|c|}{} \\
\hline \multicolumn{5}{|c|}{$\overline{N P V}=\frac{500}{(1+.05)}+\frac{500}{(1+.05)^{2}}-500=430$} \\
\hline
\end{tabular}


Para el proyecto propuesto se calcula la desviación típica con las diferentes probabilidades de cada flujo de efectivo. El cálculo se muestra a continuación.

\section{Cuadro 6.}

\begin{tabular}{|c|c|c|c|c|c|c|c|}
\hline \multicolumn{8}{|c|}{ Desviación Estándar } \\
\hline \multicolumn{4}{|c|}{ Año 1} & \multicolumn{4}{|c|}{ Año 2} \\
\hline$p$ & $(R-\bar{R})$ & $(R-\bar{R})^{2}$ & $(R-\bar{R})^{2} p$ & $p$ & $(R-\bar{R})$ & $(R-\bar{R})^{2}$ & $(R-\bar{R})^{2} p$ \\
\hline .2 & -200 & 40000 & 8000 & .25 & -100 & 10000 & 2500 \\
\hline .6 & 0 & 0 & 0 & .50 & 0 & 0 & 0 \\
\hline \multirow[t]{2}{*}{.2} & 200 & 40000 & 8000 & .25 & 100 & 10000 & 2500 \\
\hline & & & 16000 & & & & 5000 \\
\hline \multicolumn{8}{|c|}{$\sigma=136$} \\
\hline
\end{tabular}

La desviación típica del valor presente es:

$$
\sigma=\sqrt{\sum_{t=1}^{n} \frac{\left(\sigma_{t}\right)^{2}}{\left(1+r_{j}\right)^{2 t}}}
$$

donde $\sigma$ es la desviación típica del NPV y el exponente del denominador, $2 t$, indica discontinuidades para $\sigma$ en los valores $\left(1+r_{f}\right)^{2}$ y $\left(1+r_{f}\right)^{4}$. Con los resultados anteriores, y construyendo nuestros intervalos, se concluye que la probabilidad es cercana a cero si el NPV es menor a $22(430-3 \sigma)$ o mayor a $838(430+3 \sigma)$. Por último, tenemos que mencionar que los cálculos anteriores son válidos cuando los flujos de efectivo son independientes a través de los años.

Por otra parte, existen dos métodos para calcular el valor presente neto ajustado bajo riesgo. Estos métodos, incorporan al riesgo en el cálculo del valor esperado, pero sin utilizar la desviación típica.

1.- El riesgo ajustado a la tasa de descuento (RADR) es aquel en el cual el ajuste se realiza sobre el denominador del cálculo del valor presente.

2.- La certeza equivalente es aquella en donde el riesgo es ajustado en el numerador del valor presente.

El método del riesgo ajustado en la tasa de descuento es el más usado. Sabemos que la tasa de descuento a la cual los flujos de capital de un proyecto es descontada en el presente, está formada por dos componentes, la tasa de menor riesgo $\left(r_{f}\right)$ y el riesgo premium, $R P$ :

$$
K=r_{f}+R P .
$$

La tasa libre de riego es, en el sentido ideal, el valor del dinero. El riesgo premiun representa un juicio de cómo compensaciones adicionales son necesarias para compensar el riesgo adicional. 
Por ejemplo, suponga que la tasa libre de riesgo es del $6 \%$, pero una corporación utiliza el costo de capital del $10 \%$ para proyectos que se considera conllevan un riesgo promedio. En este caso, el riesgo premium es del $4 \%$. Sin embargo, esto no significa que todas los departamentos o divisiones de la compañía siempre utilicen el $10 \%$ como tasa de descuento.

Una compañía está conformada por divisiones con diferentes niveles de riesgo. Supongamos una empresa con dos divisiones de tamaño similar: la división de seguridad (suponga un supermercado) y la división de riesgo (alta tecnología en operación). Si estas dos divisiones se consideran como compañías separadas, el riesgo ajustado en la tasa de descuento es del $8 \%$ y $12 \%$ respectivamente, las cuales son diferentes a la tasa del 10\%. Por otra parte, consideremos el caso en que la división de riesgo está analizando dos proyectos: el desarrollo de un nuevo producto y el reemplazamiento de algún equipo ineficiente. Obviamente, la compañía deberá descontar con una tasa más alta al primero que al último proyecto, ya que tienen diferente nivel de riesgo. ${ }^{2}$

En el cálculo del riesgo ajustando las tasas de descuento, la incorporación del riesgo se obtiene alterando la tasa de descuento o el valor del capital en el denominador de la ecuación de descuento. En otras palabras, los flujos de efectivo descontados pueden ser expresados de la siguiente forma, en donde el riesgo es incluído en la fórmula haciendo crecer el denominador.

$$
P V=\sum_{t=1}^{n} \frac{R_{t}}{\left(1+r_{t}+R_{p}\right)^{t}}
$$

Otra técnica para incluir el riesgo en el cálculo del valor presente es trabajando en el numerador de la fracción de los flujos de efectivo. Esto es, los flujos de efectivo mismos son ajustados considerando el riesgo. Básicamente esto es realizado aplicando un factor a cada flujo de efectivo para convertir un flujo de alto riesgo en otro menos riesgoso. Ya que las empresas son adversas al riesgo, prefieren esperar pequeños flujos de efectivo libres de riesgo sobre aquellos que se encuentran en condiciones de riesgo. Para conseguir esto, los flujos de efectivo riesgosos deben ser reducidos por la multiplicación de un factor menor que uno. Este factor de ajuste es llamado factor de certeza equivalente.

Sin embargo, el valor del factor de certeza equivalente depende de la actitud respecto al riesgo de cada empresa. De esta manera, si se decide que un flujo de efectivo es riesgoso (o el valor esperado del flujo de efectivo es riesgoso), por ejemplo, $\$ 500.00$ es equivalente a un flujo de efectivo libre de riesgo de $\$ 450.00$ y el factor de certeza equivalente $\left(a_{t}\right)$ es igual a $0.9:(0.9)(500)=450$.

Así para cada flujo de efectivo riesgoso, $R_{t}$, un factor de certeza equivalente es asignado. Si el riesgo crece en función del tiempo, el factor de certeza equivalente decrece al futuro. Por ejemplo, un proyecto tiene los siguientes flujos de efectivo y factores de certeza equivalente:

2 Estudios recientes muestran que alrededor del $70 \%$ de las compañías miden el riesgo en proyectos o grupos de proyectos agrupándolos según diferentes tipos de riesgo. 
Cuadro 7 .

\begin{tabular}{|cccc|}
\hline Periodo & $R_{t}$ & $a_{t}$ & $\left(a_{t}\right)\left(R_{t}\right)$ \\
\hline 1 & 100 & 0.95 & 95 \\
2 & 200 & 0.90 & 180 \\
3 & 200 & 0.85 & 170 \\
4 & 100 & 0.80 & 80 \\
\hline
\end{tabular}

Los flujos de efectivo libres de riesgo $\left(a_{t} R_{t}\right)$ son obviamente menores que los flujos de efectivo riesgosos, como esperaría una persona adversa al riesgo. Así, los flujos de efectivo son descontados por una tasa libre de riesgo para obtener el valor presente de los flujos de efectivo.

\section{Conclusiones}

Los métodos que consideran el riesgo sin el cálculo específico de la desviación típica han sido desarrollados, pero ¿Cuál de los dos métodos es preferible? La técnica de certeza equivalente es más sofisticada y a veces es recomendada por los académicos, aunque las empresas prefieren el método RADR. La razón de esta preferencia obedece a que es más fácil realizar una estimación del costo de capital que calcular el factor de certeza equivalente para cada flujo de efectivo. Sin embargo, se puede demostrar que ambos métodos llegan a los mismos resultados si los cálculos y las suposiciones son realizados adecuadamente. El valor presente de un flujo de efectivo riesgoso descontado puede ser escrito de la siguiente manera:

$$
\frac{R_{i}}{(1+k)^{t}}
$$

Por otro lado, el valor presente de un flujo de efectivo descontado por una tasa libre de riesgo es:

$$
\frac{a_{t} R_{t}}{\left(1+r_{f}\right)^{t}} \text {. }
$$

Si ambos son iguales, entonces,

$$
\begin{gathered}
\frac{R_{t}}{(1+k)^{t}}=\frac{a_{t} R_{t}}{\left(1+r_{f}\right)^{t}} \\
a_{t}=\frac{\left(1+r_{f}\right)^{t}}{(1+k)^{t}}
\end{gathered}
$$

En esta solución, $\left(a_{t}\right)$ decrece a través del tiempo, mientras que $(k)$ permanece constante. Esto implica que para una constante $(k)$, el riesgo crece a través del tiempo. Si este es el caso, entonces es apropiado utilizar $(k)$ como constante. Sin embargo, en ocasiones el riesgo no crece respecto al tiempo, entonces el analista deberá estar consiente que usar una constante RADR puede penalizar gran número de términos de un proyecto que en efecto no son afectados por riesgos altos. 


\section{Bibliografía}

Bill, R. (1996). Financial Analisis. Prentice Hall PTR.

Danthine, J.P. and J. Donaldson (2002). Intermediate Financial Therry. Prentice Hall.

Bodie, Z. and R. C. Merton (2000). Finance. Prentice Hall.

Shimell, P. (2002). The Universe of Risk: How top Business Leaders Control Risk and Achieve Success. Prentice Hall. 